\title{
A NOTE ON THE VIRTUAL WAITING TIME IN THE STATIONARY PH/M/c+D QUEUE
}

\author{
KEN'ICHI KAWANISHI,* Gunma University \\ TETSUYA TAKINE,** Osaka University
}

\begin{abstract}
In this paper we consider the stationary $\mathrm{PH} / \mathrm{M} / \mathrm{c}$ queue with deterministic impatience times $(\mathrm{PH} / \mathrm{M} / \mathrm{c}+\mathrm{D})$. We show that the probability density function of the virtual waiting time takes the form of a matrix exponential whose exponent is given explicitly by system parameters.

Keywords: $\mathrm{PH} / \mathrm{M} / \mathrm{c}$ queue; deterministic impatience time; virtual waiting time; matrix exponential form

2010 Mathematics Subject Classification: Primary 60K25
\end{abstract}

Secondary 68M20

\section{Introduction}

In this paper we consider a stationary $\mathrm{PH} / \mathrm{M} / \mathrm{c}$ queue with deterministic impatience times (i.e. the $\mathrm{PH} / \mathrm{M} / \mathrm{c}+\mathrm{D}$ queue). Customers arrive according to a phase-type renewal process whose renewal intervals follow a phase-type distribution with representation $(\boldsymbol{\alpha}, \boldsymbol{C})$, where $\boldsymbol{\alpha}$ and $\boldsymbol{C}$ denote a $1 \times m$ vector and an $m \times m$ matrix. We assume that $\boldsymbol{\alpha} \boldsymbol{e}=1$ and $\boldsymbol{C}+(-\boldsymbol{C}) \boldsymbol{e} \boldsymbol{\alpha}$ is irreducible, where $\boldsymbol{e}$ denotes an $m \times 1$ vector with $1 \mathrm{~s}$. All customers have a deterministic waiting time limit $\tau>0$, which we call the impatience time, and if their waiting times exceed $\tau$, customers leave the system without receiving their services. There are $c$ servers and customers are served on a first-come-first-served (FCFS) basis. Service times of customers are independent and identically distributed according to an exponential distribution with mean $c / \mu$. We assume that the system is stationary.

Multi-server queues with non-Poisson arrivals and deterministic impatience times were studied in [1] and [3]. In [3], the Cox/M/c+D queue was studied and the spectral analysis of the joint distribution of workload assigned to each server was carried out. In [1], the MAP/M/c+D queue was studied, where customers arrive according to a Markovian arrival process (MAP) characterized by $(\boldsymbol{C}, \boldsymbol{D})$. Note that the phase-type renewal arrival process is a special case of a MAP, where $\boldsymbol{D}=(-\boldsymbol{C}) \boldsymbol{e} \boldsymbol{\alpha}$ and, therefore, the results for the MAP/M/c+D queue are directly applicable to our model. We thus summarize the results of [1].

Let $L, V$, and $S$ denote the number of customers, the virtual waiting time, and the state of the phase-type distribution, respectively, in steady state. Note that the virtual waiting time is regarded as the actual waiting time of a randomly arriving (virtual) customer with no waiting time limit. Let $\boldsymbol{q}_{k}(k=0,1, \ldots, c-1)$ denote a $1 \times m$ vector whose $j$ th $(j=1,2, \ldots, m)$

Received 19 August 2014; revision received 1 October 2014.

* Postal address: Division of Electronics and Informatics, Gunma University, Kiryu, 376-8515, Japan.

Email address: kawanisi@cs.gunma-u.ac.jp

** Postal address: Department of Information and Communications Technology, Graduate School of Engineering, Osaka University, Suita, 565-0871, Japan. Email address: takine@ comm.eng.osaka-u.ac.jp 
element represents $\mathbb{P}(L=k, S=j)$. We define $\boldsymbol{v}(x)(x>0)$ as the probability density function (PDF) of $V$.

Let $\boldsymbol{B}_{k}(k=0,1, \ldots, c-1)$ denote an $m \times m$ matrix determined recursively by $\boldsymbol{B}_{0}=\boldsymbol{C}$ and

$$
\boldsymbol{B}_{k}=\boldsymbol{C}-\frac{k \mu}{c} \boldsymbol{I}+\frac{k \mu}{c}\left(-\boldsymbol{B}_{k-1}\right)^{-1} \boldsymbol{D} \text { for } k=1,2, \ldots, c-1,
$$

where $\boldsymbol{I}$ denotes a unit matrix. Note that $\boldsymbol{B}_{k}(k=0,1, \ldots, c-1)$ can be regarded as a defective generator of a Markov chain (cf. Lemma 1 in Section 2) and, therefore, it is nonsingular. From (2.8), (2.14), and (2.15), and Theorems 1 and 2 of [1], it follows that

$$
\begin{gathered}
\boldsymbol{v}(x)=\boldsymbol{v}(\tau-) \exp [-\mu(x-\tau)] \quad \text { for } x \geq \tau, \\
\frac{\mathrm{d}^{2}}{\mathrm{~d} x^{2}} \boldsymbol{v}(x)+\frac{\mathrm{d}}{\mathrm{d} x} \boldsymbol{v}(x)(\mu \boldsymbol{I}+\boldsymbol{C})+\mu \boldsymbol{v}(x)(\boldsymbol{C}+\boldsymbol{D})=\mathbf{0} \text { for } 0<x<\tau, \\
\left.\frac{\mathrm{d}}{\mathrm{d} x} \boldsymbol{v}(x)\right|_{x=0+}=-\boldsymbol{v}(0+)\left(\boldsymbol{C}+\mu\left(-\boldsymbol{B}_{c-1}\right)^{-1} \boldsymbol{D}\right), \\
\left.\frac{\mathrm{d}}{\mathrm{d} x} \boldsymbol{v}(x)\right|_{x=\tau-}=\boldsymbol{v}(\tau-)(\boldsymbol{D}-\mu \boldsymbol{I}), \\
\boldsymbol{q}_{k}=\frac{1}{\mu} \boldsymbol{v}(0+) \boldsymbol{A}_{c-1} \boldsymbol{A}_{c-2} \cdots \boldsymbol{A}_{k} \quad \text { for } k=0,1, \ldots, c-1,
\end{gathered}
$$

where $\boldsymbol{A}_{k}$ for $k=0,1, \ldots, c-1$ is given by

$$
\boldsymbol{A}_{k}=\frac{(k+1) \mu}{c}\left(-\boldsymbol{B}_{k}\right)^{-1} \text {. }
$$

In [1], the authors carefully examine the second-order differential equation (3) and obtain its solution, which takes the form of a linear combination of two matrix exponentials whose exponents are given by solutions of matrix quadratic equations.

The purpose of this paper is to show that for the special case of phase-type renewal arrivals, i.e. $\boldsymbol{D}=(-\boldsymbol{C}) \boldsymbol{e} \boldsymbol{\alpha}$, the PDF $\boldsymbol{v}(x)$ for $0<x<\tau$ of the stationary virtual waiting time takes a matrix-exponential form whose exponent is given explicitly by system parameters.

Theorem 1. In the $\mathrm{PH} / \mathrm{M} / \mathrm{c}+\mathrm{D}$ queue, $\boldsymbol{v}(x)$ for $0<x<\tau$ is given by

$$
\boldsymbol{v}(x)=p \hat{\boldsymbol{v}} \exp [(\mu \boldsymbol{e} \boldsymbol{\alpha}+\boldsymbol{C})(\tau-x)] \text { for } 0<x<\tau,
$$

where the $1 \times m$ positive boundary vector $\hat{\boldsymbol{v}}:=\boldsymbol{v}(\tau-) /(\boldsymbol{v}(\tau-) \boldsymbol{e})$ is given by

$$
\hat{\boldsymbol{v}}=\frac{\boldsymbol{\alpha}(\mu \boldsymbol{I}-\boldsymbol{C})^{-1}}{\boldsymbol{\alpha}(\mu \boldsymbol{I}-\boldsymbol{C})^{-1} \boldsymbol{e}}
$$

and with $\hat{\boldsymbol{q}}_{k}:=\boldsymbol{q}_{k} /(\boldsymbol{v}(\tau-) \boldsymbol{e})$ for $k=0,1, \ldots, c-1$ defined as

$$
\hat{\boldsymbol{q}}_{k}=\frac{1}{\mu} \hat{\boldsymbol{v}} \exp [(\mu \boldsymbol{e} \boldsymbol{\alpha}+\boldsymbol{C}) \tau] \boldsymbol{A}_{c-1} \boldsymbol{A}_{c-2} \cdots \boldsymbol{A}_{k} .
$$

The normalizing constant $p:=\boldsymbol{v}(\tau-) \boldsymbol{e}$ is given by

$$
p=\left[\sum_{k=0}^{c-1} \hat{\boldsymbol{q}}_{k} \boldsymbol{e}+\frac{1}{\mu}+\left(\begin{array}{ll}
1 & \mathbf{0}
\end{array}\right) \exp \left[\left(\begin{array}{lc}
0 & \hat{\boldsymbol{v}} \\
\mathbf{0} & \mu \boldsymbol{e} \boldsymbol{\alpha}+\boldsymbol{C}
\end{array}\right) \tau\right]\left(\begin{array}{l}
0 \\
\boldsymbol{e}
\end{array}\right)\right]^{-1} .
$$

We prove Theorem 1 in Section 2 and concluding remarks are provided in Section 3. 


\section{Proof of Theorem 1}

We start with the following lemma.

Lemma 1. It holds that $\boldsymbol{B}_{k}$ in (1) satisfies

$$
\left(-\boldsymbol{B}_{k}\right)^{-1}(-\boldsymbol{C}) \boldsymbol{e}=\boldsymbol{e} \text { for } k=0,1, \ldots, c-1 .
$$

Remark 1. Lemma 1 implies that (1) for the $\mathrm{PH} / \mathrm{M} / \mathrm{c}+\mathrm{D}$ queue with $\boldsymbol{D}=(-\boldsymbol{C}) \boldsymbol{e} \boldsymbol{\alpha}$ can be written as

$$
\boldsymbol{B}_{k}=\boldsymbol{C}+\frac{k \mu}{c}(\boldsymbol{e} \boldsymbol{\alpha}-\boldsymbol{I}) \quad \text { for } k=1,2, \ldots, c-1 .
$$

Proof of Lemma 1. We prove Lemma 1 by induction. By definition $\left(-\boldsymbol{B}_{0}\right)^{-1}(-\boldsymbol{C}) \boldsymbol{e}=\boldsymbol{e}$. Suppose that (11) holds for some $k-1$ for $k=1, \ldots, c-1$. Post-multiplying both sides of (1) by $\boldsymbol{e}$ and noting that $\boldsymbol{D} \boldsymbol{e}=(-\boldsymbol{C}) \boldsymbol{e}$ yields

$$
\boldsymbol{B}_{k} \boldsymbol{e}=\boldsymbol{C} \boldsymbol{e}-\frac{k \mu}{c}\left[\boldsymbol{e}-\left(-\boldsymbol{B}_{k-1}\right)^{-1}(-\boldsymbol{C}) \boldsymbol{e}\right]=\boldsymbol{C e},
$$

from which we obtain $\left(-\boldsymbol{B}_{k}\right)^{-1}(-\boldsymbol{C}) \boldsymbol{e}=\boldsymbol{e}$.

Proof of Theorem 1. We define $\boldsymbol{f}(x)$ as

$$
\boldsymbol{f}(x)=\frac{\mathrm{d}}{\mathrm{d} x} \boldsymbol{v}(x)+\boldsymbol{v}(x)(\mu \boldsymbol{e} \boldsymbol{\alpha}+\boldsymbol{C}) \text { for } 0<x<\tau .
$$

From (3), (12), and $\boldsymbol{\alpha} \boldsymbol{e}=1$, it follows that

$$
\begin{aligned}
\frac{\mathrm{d}}{\mathrm{d} x} \boldsymbol{f}(x) & =\frac{\mathrm{d}^{2}}{\mathrm{~d} x^{2}} \boldsymbol{v}(x)+\frac{\mathrm{d}}{\mathrm{d} x} \boldsymbol{v}(x)(\mu \boldsymbol{e} \boldsymbol{\alpha}+\boldsymbol{C}) \\
& =-\left[\frac{\mathrm{d}}{\mathrm{d} x} \boldsymbol{v}(x)(\mu \boldsymbol{I}+\boldsymbol{C})+\mu \boldsymbol{v}(x)(\boldsymbol{C}+(-\boldsymbol{C}) \boldsymbol{e} \boldsymbol{\alpha})\right]+\frac{\mathrm{d}}{\mathrm{d} x} \boldsymbol{v}(x)(\mu \boldsymbol{e} \boldsymbol{\alpha}+\boldsymbol{C}) \\
& =\mu \frac{\mathrm{d}}{\mathrm{d} x} \boldsymbol{v}(x)(\boldsymbol{e} \boldsymbol{\alpha}-\boldsymbol{I})-\mu \boldsymbol{v}(x)(\boldsymbol{C}+(-\boldsymbol{C}) \boldsymbol{e} \boldsymbol{\alpha}) \\
& =\mu[\boldsymbol{f}(x)-\boldsymbol{v}(x)(\mu \boldsymbol{e} \boldsymbol{\alpha}+\boldsymbol{C})](\boldsymbol{e} \boldsymbol{\alpha}-\boldsymbol{I})-\mu \boldsymbol{v}(x)[\boldsymbol{C}+(-\boldsymbol{C}) \boldsymbol{e} \boldsymbol{\alpha}] \\
& =\mu \boldsymbol{f}(x)(\boldsymbol{e} \boldsymbol{\alpha}-\boldsymbol{I})
\end{aligned}
$$

and, therefore,

$$
\boldsymbol{f}(x)=\boldsymbol{f}(0+) \exp [\mu(\boldsymbol{e} \boldsymbol{\alpha}-\boldsymbol{I}) x] \text { for } 0<x<\tau .
$$

Next we consider $\boldsymbol{f}(0+)$. From (4) and (12), it follows that

$$
\boldsymbol{f}(0+)=\left.\frac{\mathrm{d}}{\mathrm{d} x} \boldsymbol{v}(x)\right|_{x=0+}+\boldsymbol{v}(0+)(\mu \boldsymbol{e} \boldsymbol{\alpha}+\boldsymbol{C})=\boldsymbol{v}(0+)\left[\mu \boldsymbol{e}-\mu\left(-\boldsymbol{B}_{c-1}\right)^{-1}(-\boldsymbol{C}) \boldsymbol{e}\right] \boldsymbol{\alpha} .
$$

Therefore, Lemma 1 implies that $\boldsymbol{f}(0+)=\mathbf{0}$, so $\boldsymbol{f}(x)=\mathbf{0}$, i.e.

$$
\frac{\mathrm{d}}{\mathrm{d} x} \boldsymbol{v}(x)=-\boldsymbol{v}(x)(\mu \boldsymbol{e} \boldsymbol{\alpha}+\boldsymbol{C}) \text { for } 0<x<\tau
$$

or, equivalently,

$$
\boldsymbol{v}(x)=\boldsymbol{v}(0+) \exp [-(\mu \boldsymbol{e} \boldsymbol{\alpha}+\boldsymbol{C}) x]=\boldsymbol{v}(\tau-) \exp [(\mu \boldsymbol{e} \boldsymbol{\alpha}+\boldsymbol{C})(\tau-x)] \text { for } 0<x<\tau .
$$

We thus have (7). 
Finally, we determine $\boldsymbol{v}(\tau-)$. From (13), it follows that

$$
\left.\frac{\mathrm{d}}{\mathrm{d} x} \boldsymbol{v}(x)\right|_{x=\tau-}=-\boldsymbol{v}(\tau-)(\mu \boldsymbol{e} \boldsymbol{\alpha}+\boldsymbol{C}) .
$$

Therefore, from (5) and (14), we obtain

$$
\boldsymbol{v}(\tau-)[\mu(\boldsymbol{e} \boldsymbol{\alpha}-\boldsymbol{I})+\boldsymbol{C}+(-\boldsymbol{C}) \boldsymbol{e \alpha}]=\mathbf{0} .
$$

Note that $\boldsymbol{A}:=\mu(\boldsymbol{e} \boldsymbol{\alpha}-\boldsymbol{I})+\boldsymbol{C}+(-\boldsymbol{C}) \boldsymbol{e} \boldsymbol{\alpha}$ can be regarded as a generator of a Markov chain. Furthermore, $\boldsymbol{C}+(-\boldsymbol{C}) \boldsymbol{e} \boldsymbol{\alpha}$ is irreducible, so is $\boldsymbol{A}$. Therefore, (15) uniquely determines $\boldsymbol{v}(\tau-)$ up to a multiplicative constant. Because $\boldsymbol{A}$ can be factored to be $\boldsymbol{A}:=(\mu \boldsymbol{I}-\boldsymbol{C})(\boldsymbol{e} \boldsymbol{\alpha}-\boldsymbol{I})$ and $\mu \boldsymbol{I}-\boldsymbol{C}$ is nonsingular, we obtain (8).

It remains to show (10). Noting that $\boldsymbol{v}(0+)=\boldsymbol{v}(\tau-) \exp [(\mu \boldsymbol{e} \boldsymbol{\alpha}+\boldsymbol{C}) \tau]$ and $\boldsymbol{v}(\tau-)=p \cdot \hat{\boldsymbol{v}}$, we write (6) as

$$
\boldsymbol{q}_{k}=\frac{1}{\mu} p \hat{\boldsymbol{v}} \exp [(\mu \boldsymbol{e} \boldsymbol{\alpha}+\boldsymbol{C}) \tau] \boldsymbol{A}_{c-1} \boldsymbol{A}_{c-2} \cdots \boldsymbol{A}_{k} .
$$

On the other hand, due to the law of total probability, we have

$$
\sum_{k=0}^{c-1} \boldsymbol{q}_{k} \boldsymbol{e}+\int_{0}^{\infty} \boldsymbol{v}(x) \boldsymbol{e} \mathrm{d} x=1 .
$$

Thus, from (2), (9), (16), and (17), we obtain

$$
p \sum_{k=0}^{c-1} \hat{\boldsymbol{q}}_{k} \boldsymbol{e}+\int_{0}^{\tau} \boldsymbol{v}(x) \boldsymbol{e} \mathrm{d} x+p \hat{\boldsymbol{v}} \boldsymbol{e} \frac{1}{\mu}=1 .
$$

Then (10) now follows from $\hat{\boldsymbol{v}} \boldsymbol{e}=1$ and

$$
\begin{aligned}
& \int_{0}^{\tau} \boldsymbol{v}(x) \boldsymbol{e} \mathrm{d} x=p \int_{0}^{\tau} \hat{\boldsymbol{v}} \exp [(\mu \boldsymbol{e} \boldsymbol{\alpha}+\boldsymbol{C})(\tau-x)] \mathrm{d} x \boldsymbol{e} \\
& =p \int_{0}^{\tau} \mathrm{e}^{0 \times x} \hat{\boldsymbol{v}} \exp [(\mu \boldsymbol{e} \boldsymbol{\alpha}+\boldsymbol{C})(\tau-x)] \mathrm{d} x \boldsymbol{e} \\
& =p\left(\begin{array}{ll}
1 & \mathbf{0}
\end{array}\right) \exp \left[\left(\begin{array}{cc}
0 & \hat{\boldsymbol{v}} \\
\mathbf{0} & \mu \boldsymbol{e} \boldsymbol{\alpha}+\boldsymbol{C}
\end{array}\right) \tau\right]\left(\begin{array}{l}
0 \\
\boldsymbol{I}
\end{array}\right) \boldsymbol{e},
\end{aligned}
$$

where the last equality follows from [5, Theorem 1].

\section{Concluding remarks}

We derived a simple formula for the PDF $v(x)$ of the virtual waiting time in the $\mathrm{PH} / \mathrm{M} / \mathrm{c}+\mathrm{D}$ queue. In [1], the authors studied the MAP/M/c+D queue, which includes the $P H / M / c+D$ queue as a special case. Therefore, the general formula for $v(x)$ in the MAP/M/c+D queue is applicable to the $\mathrm{PH} / \mathrm{M} / \mathrm{c}+\mathrm{D}$ queue. Note that the general formula in [1] is given by a linear combination of two matrix exponentials whose coefficient vectors may have negative elements. Therefore, it may not be suitable for numerical computation.

On the other hand, our formula is numerically feasible. In computing $\boldsymbol{v}(x)$ in Theorem 1 , we have to compute matrix exponentials. Note that their exponents are ML-matrices, i.e. matrices 
whose off-diagonal elements are nonnegative. Matrix exponential $\exp [\boldsymbol{A} x]$ with an $m \times m$ ML-matrix $\boldsymbol{A}$ can be computed as follows; see [2]. Let $\theta$ denote $\theta=\max _{1 \leq i \leq m}\left([\boldsymbol{A e}]_{i}\right)$. If $\theta \leq 0, \boldsymbol{A}$ can be regarded as a (defective) generator of a Markov chain and, therefore, the uniformization technique [4] is directly applicable. On the other hand, if $\theta>0$, we write $\exp [\boldsymbol{A} x]$ to be $\exp (\theta x) \exp [(\boldsymbol{A}-\theta \boldsymbol{I}) x]$. Note that $\boldsymbol{A}-\theta \boldsymbol{I}$ can be regarded as a (defective) generator of a Markov chain, so that the uniformization technique is applicable to it. This is the reason why we chose the boundary vector at $x=\tau-$, rather than $x=0+(\mathrm{cf}$. (13)).

\section{Acknowledgement}

This research was supported in part by a Grant-in-Aid for Scientific Research (C) of the Japan Society for the Promotion of Science (grant no. 25330027 and 26350416).

\section{References}

[1] Choi, B. D., Kim, B. And Zhu, D. (2004). MAP/M/c queue with constant impatient time. Math. Operat. Res. 29, 309-325.

[2] Sakuma, Y. and Takine, T. (2015). Multi-class M/PH/1 queues with deterministic impatience times. Submitted.

[3] Swensen, A. R. (1986). On a GI/M/c queue with bounded waiting times. Operat. Res. 34, 895-908.

[4] TiJms, H. C. (1994). Stochastic Models: An Algorithmic Approach, John Wiley, Chichester.

[5] VAn LoAn, C. F. (1978). Computing integrals involving the matrix exponential. IEEE Trans. Automatic Control 23, 395-404. 